BMJ Open

Diabetes

Research

\& Care

\title{
Effects of a multifactorial ecosustainable isocaloric diet on liver fat in patients with type 2 diabetes: randomized clinical trial
}

Giuseppe Della Pepa, ${ }^{1}$ Claudia Vetrani, ${ }^{1}$ Valentina Brancato, ${ }^{2}$ Marilena Vitale, ${ }^{1}$ Serena Monti, ${ }^{3}$ Giovanni Annuzzi, ${ }^{1}$ Gianluca Lombardi, ${ }^{1}$ Anna Izzo, ${ }^{1}$ Marianna Tommasone, ${ }^{1}$ Paola Cipriano, ${ }^{1}$ Gennaro Clemente, ${ }^{4}$ Peppino Mirabelli, ${ }^{2}$ Marcello Mancini, ${ }^{3}$ Marco Salvatore (D) , ${ }^{2}$ Gabriele Riccardi, ${ }^{1}$ Angela Albarosa Rivellese (D) , ${ }^{1}$ Lutgarda Bozzetto ${ }^{1}$

To cite: Della Pepa G, Vetrani C, Brancato $\mathrm{V}$, et al. Effects of a multifactorial ecosustainable isocaloric diet on liver fat in patients with type 2 diabetes: randomized clinical trial. BMJ Open Diab Res Care 2020;8:e001342. doi:10.1136/ bmjdrc-2020-001342

- Additional material is published online only. To view, please visit the journal online (http://dx.doi.org/10.1136/ bmjdrc-2020-001342).

GDP and CV contributed equally.

GDP and $\mathrm{CV}$ are joint first authors.

Received 9 March 2020 Revised 11 April 2020 Accepted 23 April 2020

Check for updates

C Author(s) (or their employer(s)) 2020. Re-use permitted under CC BY-NC. No commercial re-use. See rights and permissions. Published by BMJ.

For numbered affiliations see end of article.

Correspondence to Dr Angela Albarosa Rivellese; rivelles@unina.it

\section{ABSTRACT}

Introduction Treatment options for non-alcoholic fatty liver disease (NAFLD) in patients with type 2 diabetes (T2D) are still a matter of debate. We compared the effects of a diet including different components versus a proven beneficial diet rich in monounsaturated fatty acids (MUFAs) on liver fat in T2D.

Research design and methods According to a parallel design, 49 individuals with T2D, overweight/obese, with high waist circumference, 35-75 years-old, in satisfactory blood glucose control with diet or drugs not affecting liver fat content, were randomly assigned to an 8-week isocaloric intervention with a MUFA diet $(n=26)$ or a multifactorial diet rich in fiber, MUFA, n-6 and n-3 polyunsaturated fatty acids, polyphenols, and vitamins $D$, $E$, and $C(n=23)$. Before and after the intervention, liver fat content was evaluated by proton magnetic resonance spectroscopy ( $\left.{ }^{1} \mathrm{H}-\mathrm{MRS}\right) .{ }^{1} \mathrm{H}-\mathrm{MRS}$ complete data were available for $n=21$ (MUFA diet) and $n=18$ (multifactorial diet) participants.

Results Adherence to dietary interventions was optimal. No significant differences between groups in body weight reduction, plasma glycated hemoglobin, insulin, glucose, lipids and liver enzymes were observed. Liver fat significantly decreased after both the multifactorial $\operatorname{diet}(9.18 \% \pm 7.78 \%$ vs $5.22 \% \pm 4.80 \%, p=0.003)$ and the MUFA diet $(9.47 \% \pm 8.89 \%$ vs $8.07 \% \pm 8.52 \%, p=0.027)$ with a statistically significant difference between changes either in absolute terms $(-4.0 \% \pm 4.5 \%$ vs $-1.4 \% \pm 2.7 \%$, $\mathrm{p}=0.035)$ or percent $(-40 \% \pm 33 \%$ vs $-19 \% \pm 25 \%$, $\mathrm{p}=0.030$ ).

Conclusions An isocaloric multifactorial diet including several beneficial dietary components induced a clinically relevant reduction of liver fat in patients with $\mathrm{T} 2 \mathrm{D}$, more pronounced than that induced by simply replacing saturated fat with MUFA. This suggests that the 'optimal diet' for NAFLD treatment in T2D should be based on synergic actions of different dietary components on multiple pathophysiological pathways.

Trial registration number NCT03380416.

\section{INTRODUCTION}

Non-alcoholic fatty liver disease (NAFLD) is an ominous condition encompassing a

\section{Significance of this study}

What is already known about this subject?

- While epidemiological evidence suggests that the intake of whole grains, legumes, and dietary fiber may be protective on liver fat, no intervention trials are available. In clinical trials, $n-6$ polyunsaturated fatty acid (PUFA) or monounsaturated fatty acid (MUFA) improved liver fat independently of body weight changes, while supplementations with vitamins or different polyphenol combinations yielded inconclusive results.

- Only two randomized trials compared the effectiveness of dietary patterns, independently of body weight changes, on non-alcoholic fatty liver disease (NAFLD) with no univocal results.

What are the new findings?

- Our trial shows for the first time that, in patients with type 2 diabetes (T2D), an isocaloric multifactorial diet including changes in different dietary components (fiber, MUFA, n-6 and n-3 PUFAs, polyphenols, and vitamins $D, E$, and $C$ ) is more effective on liver fat than a MUFA-rich diet already proven to be effective.

- The effect size of liver fat reduction achieved with our multifactorial diet is the highest obtained so far with any dietary or pharmacological intervention for treating liver steatosis in T2D.

- Our innovative approach-isocaloric and based on small variations in the habitual diet-would be more feasible in the long term than marked modifications in energy or single nutrient intake.

wide range of histopathological and clinical pictures, from isolated steatosis (hepatic triglyceride accumulation with minimal or no inflammation) to non-alcoholic steatohepatitis $\mathrm{NASH}$, steatosis with inflammation and necrosis), and eventually cirrhosis and/or hepatocellular carcinoma (HCC).

NAFLD is highly prevalent ( $57 \%$ to $80 \%)$ in patients with type 2 diabetes (T2D), carrying 


\section{Significance of this study}

How might these results change the focus of research or clinical practice?

- Our results are clinically relevant, suggesting that the multifactorial diet could be currently considered the optimal dietary approach to prevent and treat NAFLD in patients with T2D.

- Enlarging alimentary choices as dietary therapeutic options for NAFLD in T2D might favor adherence to healthy dietary plans also in every-day life and in different social, cultural, and geographical contexts.

a higher likelihood of progression to NASH, cirrhosis and HCC and a higher mortality for all causes and cardiovascular disease. This legitimates NAFLD as a new complication of diabetes. ${ }^{12}$ Despite its morbidity burden, there are no drugs currently approved for the treatment of this condition. ${ }^{2}$ Furthermore, concerns relate to the efficacy of dietary treatment. Body weight loss has been consistently shown to reduce hepatic fat content and to improve liver histology. ${ }^{2}$ These changes are appreciable with a $3 \%-5 \%$ wt loss and increase linearly with further loss. ${ }^{34}$ Unfortunately, body weight variations are difficult to achieve and maintain. Therefore, lifestyle modifications able to improve liver health independently of weight changes are highly warranted, especially in patients at high risk of NAFLD, such as patients with T2D.

There are indications that isocaloric dietary changes may influence liver fat content and that the quality of macronutrients impacts liver steatosis as much as their quantity. ${ }^{5}$ In general, replacing total carbohydrates with an isoenergetic amount of total fat worsens liver steatosis. ${ }^{6-8}$ However, not all carbohydrates and fats behave the same. Substituting saturated fatty acids (SFAs) with monounsaturated fatty acids (MUFAs) or $\mathrm{n}-6$ polyunsaturated fatty acids (PUFAs) induced a clinically relevant reduction of liver fat in intervention trials on different populations. ${ }^{9-11}$ As for carbohydrate quality, epidemiological evidence and intervention trials highlight the detrimental effects of simple sugars, ${ }^{12}$ in particular fructose, ${ }^{13}$ while epidemiological data show a protective action of low-glycemic index, fiber-rich foods on liver fat accumulation. ${ }^{14} 15$ Other dietary components, such as polyphenols, vitamins $\mathrm{C}, \mathrm{E}$ and $\mathrm{D}$, and n-3 PUFA, are supposed to improve NAFLD because of their antioxidant and anti-inflammatory properties, both shown in in vitro and in animal studies. ${ }^{16}$ In addition, trials in people at high cardiovascular risk have shown that diets naturally rich in polyphenols are able to beneficially affect some of the metabolic pathways involved in the pathogenesis of NAFLD, such as postprandial lipemia, oxidative stress, and glucose metabolism. ${ }^{17}$ However, trials exploring the effects on liver steatosis and fibrosis of supplementation with polyphenols and different vitamins have provided non-conclusive results, ${ }^{5}$ with no studies specifically evaluating vitamins and polyphenols coming from natural foods. Moreover, there is no evidence of the effects of combining all the aforementioned dietary components. This may be relevant, because the different dietary components, either alone or synergistically, could act on multiple mechanisms possibly leading to NAFLD, such as de novo lipogenesis, fat oxidation, inflammation, oxidative stress, and/or gut microbiota changes. Limited data from observational studies show a direct association between liver steatosis and a Western dietary pattern, and an inverse association with the Mediterranean diet adherence score.$^{19}$ However, to the best of our knowledge, only two randomized trials have compared the effectiveness of dietary patterns, including some of the aforementioned components, independently of body weight changes, on NAFLD, with no univocal results. ${ }^{20} 21$

With this in mind, we questioned whether an isocaloric diet formulated with high contents of several potentially beneficial components may be more favorable to liver fat content than an isocaloric diet naturally rich only in MUFA.

To test this hypothesis, we compared the effects on liver fat of an isocaloric multifactorial diet characterized by high contents of fiber, MUFA, n- 6 and n-3 PUFAs, polyphenols, vitamin $\mathrm{D}, \mathrm{E}$, and $\mathrm{C}$, with an isocaloric diet rich in MUFA, already shown to be effective in reducing liver fat in patients with T2D. ${ }^{9}$

\section{RESEARCH DESIGN AND METHODS \\ Participants}

Eligible patients at screening were men and postmenopausal women (35-75 years) with T2D, overweight/ obesity (body mass index (BMI) $27-35 \mathrm{~kg} / \mathrm{m}^{2}$ ), high waist circumference (men: $\geq 102 \mathrm{~cm}$, women: $\geq 88 \mathrm{~cm}$ ), good metabolic control (glycated hemoglobin $\mathrm{A}_{1 \mathrm{c}}$ $\left(\mathrm{HbA}_{1 \mathrm{c}}\right)$ levels $\leq 7.5 \%(58.5 \mathrm{mmol} / \mathrm{mol})$, fasting plasma low-density lipoprotein (LDL) cholesterol $\leq 3.36 \mathrm{mmol} / \mathrm{L}$ and triglyceride $\leq 3.95 \mathrm{mmol} / \mathrm{L}$ ), stable treatment (no changes in the previous 6 months) with diet alone or diet plus oral glucose-lowering (metformin, repaglinide, dipeptidyl peptidase-4 (DPP-4) inhibitors, or sulfonylureas), lipid-lowering (statins and ezetimibe) or antihypertensive drugs.

The exclusion criteria were the following: any acute or chronic hepatic disease, excluding NAFLD; history of alcohol intake exceeding $30 \mathrm{~g} /$ day in men and $20 \mathrm{~g}$ / day in women; any other acute/chronic disease severely affecting health status; unstable body weight $( \pm 3 \mathrm{~kg}$ changes) in the previous 6 months; current smoking; unstable food habits; regular moderate to strenuous physical activity; and supplementation with vitamins, nutraceuticals, or antioxidants.

\section{Study design}

We performed a monocentric, two-arm, open-label, randomized, controlled trial in patients with T2D regularly attending the diabetes outpatient clinic of the Federico II University Teaching Hospital (Naples, Italy).

This study was registered at ClinicalTrials.gov. 
Table 1 Composition of the diets randomly assigned and followed by the participants completing the trial

\begin{tabular}{|c|c|c|c|c|}
\hline & MUFA diet & & Multifacto & \\
\hline & Assigned & Followed* & Assigned & Followed* $^{*}$ \\
\hline Energy (kcal/day) & 2000 & 1967 (297) & 2000 & 1940 (334) \\
\hline Protein (\% TEI) & 18 & $17.6(1.7)$ & 18 & $17.8(1.2)$ \\
\hline Fat (\% TEI) & 41 & $40.9(4.0)$ & 41 & $43.1(3.6)$ \\
\hline Saturated (\% TEI) & 7 & $7.2(0.9)$ & 6 & $7.2(0.9)$ \\
\hline Monounsaturated (\% TEI) & 28 & $26.5(3.2)$ & 26 & $26.7(2.0)$ \\
\hline Polyunsaturated (\% TEI) & 4 & $4.2(0.28)$ & 6 & $5.8(0.5)^{\star}$ \\
\hline $\mathrm{n}-3(\%$ TEI) & 0.5 & $0.6(0.06)$ & 1.2 & $1.2(0.2)^{\star}$ \\
\hline n-6 (\% TEI) & 3.4 & $3.6(0.3)$ & 4.4 & $4.5(0.3)^{\star}$ \\
\hline Cholesterol (mg/day) & 187 & $175(34)$ & 122 & $113(27)^{\star}$ \\
\hline Carbohydrates (\% TEI) & 41 & $41.5(2.6)$ & 41 & $38.9(3.8)$ \\
\hline Sugars (\% TEI) & 11 & $11.0(1.4)$ & 10 & $10.0(1.5)$ \\
\hline Fiber (g/1000 kcal) & 10 & $9.3(1.1)$ & 24 & $20.5(3.8)^{\star}$ \\
\hline Glycemic index (\%) & 62 & $61(4)$ & 51 & $52(3)^{\star}$ \\
\hline Glycemic load & 127 & $121(18)$ & 95 & $102(25)^{\star}$ \\
\hline Polyphenols (mg/day) & 381 & $393(38)$ & 2747 & $2664(367)^{\star}$ \\
\hline Vitamin E (mg/day) & 22 & $20(4)$ & 24 & $23(4)^{\star}$ \\
\hline Vitamin D ( $\mu \mathrm{g} /$ day $)$ & 1 & $1.4(0.9)$ & 5 & $6.6(2.2)^{\star}$ \\
\hline Vitamin C (mg/day) & 89 & $87(25)$ & 257 & $210(67)^{\star}$ \\
\hline
\end{tabular}

${ }^{*} \mathrm{P}<0.05$ vs MUFA.

*Mean (SD) of the 7-day food records completed at weeks 4 and 8.

MUFA, monounsaturated fatty acid; TEl, total energy intake.

During a 3-week run-in period, participants were stabilized on a diet reproducing their habitual dietary habits, which are within the dietary pattern of patients with T2D in Italy ${ }^{22}$ (protein, $16 \%$ of total energy intake (TEI); fat, $33 \%$; SFA, 12\%; MUFA, 16\%; PUFA, 5\%; carbohydrate, $51 \%$; sugar, $15 \%$; fiber, $9 \mathrm{~g} / 1000 \mathrm{kcal}$; polyphenols, $360 \mathrm{mg} /$ day). At baseline, before the intervention diet, composition was similar in the two dietary intervention groups (online supplementary table 1). Participants were asked to keep their habitual physical activity unchanged during the whole experimental period. Drugs for diabetes, hypertension and lipids were not changed during the trial.

Then, participants were randomly allocated (1:1) to one of the two 8-week dietary interventions, the MUFA diet or the multifactorial diet, by a researcher not directly involved in the trial using the minimization method with MINIM software (www.users.york.ac.uk), stratified for sex, BMI $\left(27-29 \mathrm{~kg} / \mathrm{m}^{2}\right.$ or $\left.30-35 \mathrm{~kg} / \mathrm{m}^{2}\right)$, and glucoselowering treatment (diet alone or diet plus metformin, or diet plus metformin in combination with the other glucose-lowering drugs allowed in the trial).

The experimental diets were isoenergetic-in order to keep the body weight constant-and had a similar amount of total fat, MUFA and carbohydrates, while differing for n-3 and n-6 PUFAs, fiber, glycemic index, polyphenols, and vitamins $\mathrm{D}, \mathrm{E}$, and $\mathrm{C}$ (table 1 ).
The main foods characterizing the two diets are listed in table 2. The multifactorial diet was based on whole grainbased products, legumes, vegetables, oranges, rocket salad, artichokes, onions, decaffeinated coffee, green tea, high-polyphenol extra-virgin olive oil (EVOO), almonds, and salmon. The MUFA diet was based on refined flourbased foods, potatoes, low-polyphenol EVOO, daily consumption of vegetables and fruits with a low content in polyphenols and fiber.

Alcohol intake was not allowed in either group throughout the study.

To improve dietary compliance, patients were seen every 2 weeks by a skilled dietitian who also made telephone calls every 2-3 days. A weekly dietary plan was provided to the participants to ensure that frequency and amount of specific foods characterizing the dietary intervention were met. Moreover, some foods were supplied to the participants for the whole duration of the study to facilitate dietary compliance: EVOO, green peas, frozen vegetables (cauliflower and zucchini), tomato sauce, beef cured meat (bresaola), decaffeinated coffee for the MUFA diet; and polyphenol-rich EVOO, legumes (ie, lentils, cannellini beans, chickpeas and green peas), whole grain pasta, frozen vegetables (broccoli, peppers, mushrooms, artichokes and endive), frozen salmon, tomato sauce, beef cured meat (bresaola), decaffeinated coffee and green tea and almonds for the multifactorial diet. 


\begin{tabular}{ll}
\hline Table 2 Foods characterizing the two dietary interventions \\
\hline MUFA diet & Multifactorial diet \\
\hline Low fiber & High fiber \\
Refined flour-based & Whole-grain products \\
products (pasta, rice, & (pasta, bread, and bran \\
bread, and rusks) & sticks) \\
Potatoes & Legumes (lentils, cannellini \\
Vegetables and fruits & beans, green peas, and \\
(zucchini, cauliflower, and & chickpeas) at least four \\
banana) & times/week \\
& Vegetables (broccoli, \\
& peppers, mushrooms, \\
& artichokes, and endive) \\
Vegetables and fruits & High polyphenol \\
(spinach and apple) & Vegetables and fruits \\
& (rocket salad, artichokes, \\
& onions, and orange) \\
& Decaffeinated coffee (three \\
& espresso cups/day) and \\
Low-polyphenol EVOO & green tea (400mL/day) \\
& High MUFA and polyphenols \\
High MUFA-polyphenol EVOO & High n-6 PUFA \\
& High n-3 PUFA \\
& Salmon (at least three \\
& times/week) \\
\hline
\end{tabular}

Vegetables: at least two portions/day.

EVOO, extra-virgin olive oil; MUFA, monounsaturated fatty acid;

PUFA, polyunsaturated fatty acid.

Adherence to the dietary intervention was evaluated by a 7-day food record filled in by the participants at 4 and 8 weeks. In both groups, participants were considered compliant if the intake of MUFA was $\geq 80 \%$ of the amount assigned. In addition, participants allocated to the multifactorial diet were considered compliant with the dietary intervention if polyphenols and fiber intakes were $\geq 80 \%$ of the amounts assigned, while participants allocated to the MUFA diet were considered compliant if polyphenols and fiber intake were not $\geq 20 \%$ of the amounts assigned.

For all other dietary components, participants were considered compliant if for each component the intake was between $\pm 20 \%$ of assigned intakes. Moreover, participants with a weight loss of $>5 \%$ were excluded.

\section{Experimental procedures}

All measurements were taken after 12 hours of overnight fast at baseline and after 8 weeks of dietary intervention. Anthropometric measurements (weight, height, and waist circumference) were taken according to standardized procedures. ${ }^{23}$ BMI was calculated as weight (in kilogram) divided by the square of height (in meter). Blood samples were collected by an antecubital vein, immediately placed on ice, centrifuged at $4^{\circ} \mathrm{C}$, separated, and then stored at $-80^{\circ} \mathrm{C}$ until analyses. Radiologists and laboratory staff were masked to the treatment assignment.

\section{Liver fat content}

Liver fat content was measured by proton magnetic resonance spectroscopy $\left({ }^{1} \mathrm{H}-\mathrm{MRS}\right)$, performed on a $3 \mathrm{~T}$ magnetic resonance scanner (dStream, Philips Healthcare, Eindhoven, Holland) equipped with the dStream Torso coil, placed on the chest of the patients, and the dStream Posterior coil, allowing for abdominal imaging. Multiplanar localizing images covering the whole liver were preliminarily acquired to position the spectroscopy voxel. A single voxel of $20 \times 20 \times 20 \mathrm{~mm}$ was placed in the right lobe, avoiding liver edges, large vessels and large bile ducts. The spectroscopy was obtained using a point-resolved spectroscopy sequence with the following parameters: Echo Time $(\mathrm{TE})=35 \mathrm{~ms}$, Repetition Time for minutes $($ TRmin $)=3000 \mathrm{~ms}, 16$ signal averages, and 1024 data points over $2000 \mathrm{~Hz}$ spectral width. MRS scan was triggered at the end of expiration using a respiratory belt to minimize breathing artifacts. Spectral analysis was carried out offline using the LCModel software (http:/ / s-provench-er.com), which fits in vivo metabolite spectra using model resonances acquired under comparable scanning conditions from multiple compounds in standard phantom solutions (10.1002/nbm.698).$^{24}$ Values of water peak (signal of water, Sw) and the sum of lipid peaks at 1.3, 0.9, and $1.6 \mathrm{ppm}$ (signal of fat, Sf) were considered for liver fat quantification. Signal decay was corrected for the different $\mathrm{T} 2$ and $\mathrm{T} 1$ decay of water and fat using mean T2 relaxation times of 30 and $52 \mathrm{~ms}$ and mean T1 relaxation time of 990 and $402 \mathrm{~ms}$ for water and fat, respectively (10.1002/jmri.25734). Finally, the Hepatic Fat Index (HFI) was calculated as HFI $(\%)=100$. $\mathrm{Sf} /(\mathrm{Sf}+\mathrm{Sw})$.

\section{Liver elastography}

Shear wave elastography (SWE) was performed using the iU22 system (Philips) with a convex broadband probe (C5-1). ${ }^{25}$ The elastography of the system (Shear Wave Point Quantification) generates shear waves inside the liver using the acoustic force of a focused ultrasonography beam. A region of interest (ROI) with a box size of $2.0 \times 1.0 \mathrm{~cm}$ was positioned within the liver parenchyma under visual control in two-dimensional B-mode at a depth of at least $2 \mathrm{~cm}$ below the liver capsule in segments 7 or 8 of the liver. The equipment listed the shear wave velocity $(\mathrm{m} / \mathrm{s})$ in the ROI, as well as the depth at which the measurement was performed. To compute tissue stiffness in kilopascal, the shear wave velocity (v) was converted into the shear modulus $G=\tau / \gamma$, in which $\tau$ is the shear stress and $\gamma$ is the shear strain based on the relationship $\mathrm{G}=\rho \mathrm{v}^{2}$, in which $\rho$ is the density of the tissue.

\section{Metabolic parameters}

Plasma cholesterol, triglyceride, and glucose concentrations were assayed by enzymatic colorimetric methods (Roche Diagnostics, Milan, Italy, and ABX Diagnostics, Montpellier, France) on an ABX Pentra 400 (HORIBA Medical, Montpellier, France). High-density lipoprotein (HDL) cholesterol was isolated from plasma by 
Table 3 Baseline characteristics of the participants completing the trial

MUFA diet $(n=22)$

Multifactorial diet $(\mathrm{n}=21)$

\begin{tabular}{lcc}
\hline Sex & & \\
Male & $12(54)$ & $13(62$ \\
\hline Female & $10(46)$ & $8(38)$ \\
\hline Age (years) & $63(5)$ & $64(7)$ \\
\hline BMI (kg/m²) & $31(3)$ & $32(4)$ \\
\hline Plasma glucose (mmol/L) & $7.2(0.9)$ & $6.8(0.8)$ \\
\hline $\left.\mathrm{HbA}_{1 \mathrm{c}} \%\right)$ & $6.6(0.6)$ & $6.6(0.5)$ \\
\hline Glucose lowering therapy & & $7(33)$ \\
\hline Diet & $5(23)$ & $9(43$ \\
\hline Metformin & $7(32)$ & $2(10)$ \\
\hline Metformin+DPP-4 inhibitors & $8(36)$ & $3(14)$ \\
\hline Metformin+repaglinide & $2(9)$ & $10(48)$ \\
\hline Other drugs & $14(64)$ & $17(81)$ \\
\hline Statin & $19(86)$ & $7(33)$ \\
\hline Antihypertensive & $5(23)$ & \\
\hline Antiplatelet &
\end{tabular}

Data are $\mathrm{n}(\%)$ or mean (SD), not statistically different between the two groups.

$\mathrm{BMI}$, body mass index; DPP-4, dipeptidyl peptidase $4 ; \mathrm{HbA}_{1 c}$, glycated hemoglobin $\mathrm{A}_{1 c}$; MUFA, monounsaturated fatty acid.

the phosphotungstic acid/magnesium chloride precipitation method. LDL cholesterol was calculated using the Friedewald formula. Plasma insulin concentrations were measured by ELISA (DIA-source ImmunoAssay S.A., Nivelles, Belgium) on a Triturus Analyzer (Diagnostic Grifols, S.A., Barcelona, Spain). The homeostatic model assessment of insulin resistance (HOMA-IR) was calculated using the following formula: fasting glucose $(\mathrm{mmol} / \mathrm{L}) \times$ fasting insulin $(\mathrm{pmol} / \mathrm{L}) / 22$.

Serum alanine aminotransferase (ALT), aspartate aminotransferase (AST), gamma-glutamyl transpeptidase $(\gamma-G T)$ activities were determined by the Vista 1500 automated System (Siemens Healthcare Diagnostics Inc., Tarrytown, NY, USA). HbA ${ }_{1 c}$ was determined by the VARIANT II (Bio-Rad, Hercules, CA, USA) highperformance liquid chromatography (HPLC) system.

\section{Outcomes}

The primary outcome was the reduction in percentage of liver fat content evaluated by ${ }^{1} \mathrm{H}-\mathrm{MRS}$ in the multifactorial diet compared with the reduction in the MUFA diet over 8 weeks.

Secondary outcomes included between the two groups differences in 8-week changes in anthropometric and metabolic parameters, and liver enzymes. Based on our previous study, ${ }^{9}$ to detect a $20 \%$ difference in liver fat content between the two groups with an $80 \%$ power at $5 \%$ significance level, assuming a $20 \%$ dropout rate, a sample size of 23 participants for each group was devised.

\section{Statistical analysis}

Data are expressed as means $\pm \mathrm{SD}$ unless otherwise stated. Baseline differences between the two groups were evaluated by independent sample t-test with correction for multiple comparisons. Within-group and beforeafter intervention differences were assessed by paired sample t-test. The effects of the two dietary interventions were tested by two-way repeated measures analysis of variance (ANOVA). Changes in liver fat content were also evaluated as percent changes [ $(8$ week values-baseline values)/baseline values $\times 100]$.

Spearman's correlation was used to determine the strength and direction of the relationships between metabolic parameters and liver fat. A two-side $p$ value of $<0.05$ was considered significant.

The statistical analysis was performed according to standard methods using SPSS software V.25 (SPSS/PC).

\section{RESULTS}

\section{Baseline characteristics of participants}

Between 4 April 2017 and 14 January 2019, 66 patients were screened for eligibility; of these, 49 met the inclusion criteria and were randomly assigned to MUFA diet $(n=26)$ or multifactorial diet $(n=23)$ (online supplementary figure 1). Their main characteristics are shown in online supplementary table 2 . Six of these patients discontinued treatment (four assigned to MUFA diet and two to multifactorial diet). A total of 43 patients completed the trial, although for 4 of them (MUFA diet $n=1$, multifactorial diet $\mathrm{n}=3$ ), data on ${ }^{1} \mathrm{H}-\mathrm{MRS}$ were not complete. Therefore, statistical analyses were performed on 39 patients (MUFA diet $\mathrm{n}=21$, multifactorial diet $\mathrm{n}=18$ ).

At baseline, participants allocated to the two dietary intervention groups were comparable for sex, age, BMI, 
(A)

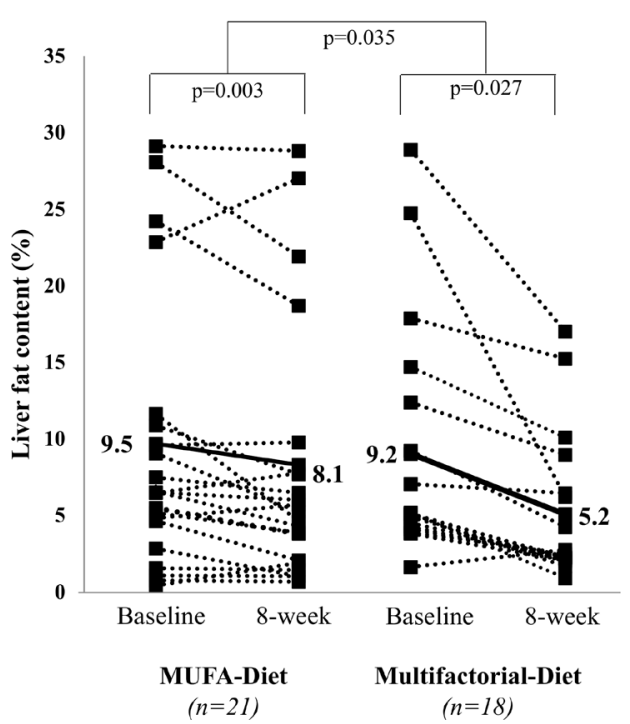

(B)

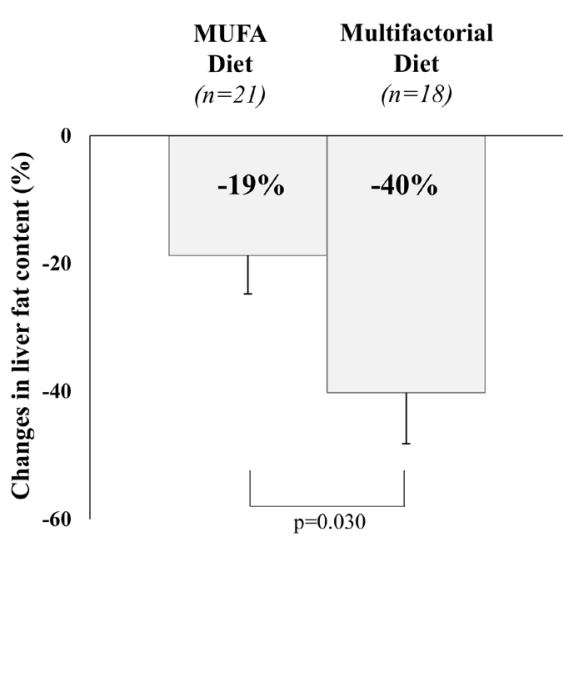

fasting plasma glucose, $\mathrm{HbA}_{1 \mathrm{c}}$ and use of glucose-lowering drugs, statins and antihypertensive drugs (table 3 ).

According to the predefined compliance criteria, one participant was excluded from the MUFA group due to low adherence to MUFA ( $<80 \%$ of the amount prescribed), and two participants were excluded from the multifactorial group, one for low adherence to MUFA, polyphenol and fiber intakes $(<80 \%$ of the amount prescribed), and the other for body weight loss higher than $5 \%$ during the intervention. Therefore, 20 patients in the MUFA diet and 16 in the multifactorial diet were included in the perprotocol analysis (online supplementary figure 1).

\section{Compliance to dietary intervention}

Overall compliance to the dietary intervention was optimal. The composition of the diets followed by the participants-average of the 7-day food records completed at weeks 4 and 8-strictly reflected the composition of the diets assigned to either group, with expected significant differences between groups in the amounts of the characterizing components (ie, n-3 and n-6 PUFAs, fiber, glycemic index, glycemic load, polyphenols, and vitamin $\mathrm{E}, \mathrm{D}$, and $\mathrm{C}$ ) (table 1 ).

\section{Liver fat content}

As for the primary endpoint, liver fat significantly decreased after both the multifactorial diet $(9.18 \% \pm 7.78 \%$ vs $5.22 \% \pm 4.80 \%, \quad \mathrm{p}=0.003)$ and the MUFA diet $(9.47 \% \pm 8.89 \%$ vs $8.07 \% \pm 8.52 \%, \mathrm{p}=0.027)$ (figure $1 \mathrm{~A})$. The liver fat reduction induced by the multifactorial diet was consistent in all participants, including the ones with hepatic fat content at baseline $<5 \%$; conversely, a less reproducible beneficial effect was observed with the MUFA diet: this was mainly confined to participants with a higher liver fat at baseline (figure 1A). The change in liver fat (8weeks minus baseline values) was significantly higher after the multifactorial diet $(-4.0 \% \pm 4.5 \%)$ than after the MUFA diet $(-1.4 \% \pm 2.7 \%),(\mathrm{p}=0.035$ by two-way repeated measures ANOVA). Percent reduction (end values-baseline $\times 100 /$ baseline) of hepatic fat was significantly greater with the multifactorial diet $(-40 \% \pm 33 \%)$ than the MUFA diet $(-19 \% \pm 25 \%)(\mathrm{p}=0.030$ by ANOVA, figure $1 \mathrm{~B})$.

Adjusting for body weight reduction did not modify the differences in liver fat changes between the two groups. Moreover, the change in liver fat after the intervention was not influenced by the type of diabetes treatment, being not statistically different among patients on (1) diet alone, (2) metformin alone, (3) metformin+DPP-4 inhibitors, and (4) metformin+repaglinide (ANOVA, $\mathrm{p}=0.808$ in the MUFA group, $\mathrm{p}=0.127$ in the multifactorial group). Similarly, the change in liver fat did not differ in statin users versus non-users $(\mathrm{p}=0.324$ in the MUFA group, $\mathrm{p}=0.833$ in the multifactorial group).

\section{Liver elastography}

We also assessed liver tissue stiffness by SWE. Tissue stiffness did not change significantly after the multifactorial diet $(6.4 \pm 3.3$ vs $5.5 \pm 1.2 \mathrm{kPa}, \mathrm{p}=0.292)$ and the MUFA diet $(5.4 \pm 1.9$ vs $5.0 \pm 1.3 \mathrm{kPa}, \mathrm{p}=0.258)$, with no significant differences between the two diets $(\mathrm{p}=0.540)$.

The presence of fibrosis was categorized based on cut-off levels known to being predictive for advanced fibrosis. ${ }^{26}$ At baseline, all participants had a low fibrosis grading ( $\mathrm{F} 0-\mathrm{F} 2$ stage, $\leq 9.54 \mathrm{kPa}$ ), apart from two participants in the multifactorial diet and one in the MUFA diet showing more advanced fibrosis (F3-F4 stage, $>9.54 \mathrm{kPa}$ ). 
Table 4 Anthropometrics and metabolic parameters of the participants completing the trial at baseline and end of intervention

\begin{tabular}{|c|c|c|c|c|c|}
\hline & \multicolumn{2}{|l|}{$\begin{array}{l}\text { MUFA diet } \\
(\mathrm{n}=22)\end{array}$} & \multicolumn{2}{|c|}{ Multifactorial diet $(n=21)$} & \multirow{2}{*}{$\begin{array}{l}\text { P value } \\
\text { diet } \times \text { time* }\end{array}$} \\
\hline & Baseline & 8 week & Baseline & 8 week & \\
\hline Body weight (kg) & $84(15)$ & $83(14) \dagger$ & $85(12)$ & $84(12) \dagger$ & 0.994 \\
\hline $\mathrm{BMI}\left(\mathrm{kg} / \mathrm{m}^{2}\right)$ & $31(3)$ & $30(3) \dagger$ & $32(4)$ & $31(4) \dagger$ & 0.894 \\
\hline Waist circumference (cm) & $104(11)$ & $104(11)$ & $106(10)$ & $106(10)$ & 0.495 \\
\hline Plasma total cholesterol (mmol/L) & $3.7(0.7)$ & $3.6(0.7)$ & $3.9(0.8)$ & $3.8(0.9)$ & 0.372 \\
\hline HDL cholesterol (mmol/L) & $1.1(0.2)$ & $1.0(0.2)$ & $1.1(0.3)$ & $1.0(0.2)$ & 0.115 \\
\hline LDL cholesterol (mmol/L) & $2.1(0.6)$ & $2.0(0.5)$ & $2.2(0.5)$ & $2.2(0.6)$ & 0.192 \\
\hline Plasma triglyceride (mmol/L) & $1.2(0.4)$ & $1.2(0.5)$ & $1.3(0.5)$ & $1.3(0.6)$ & 0.496 \\
\hline Plasma glucose (mmol/L) & $7.2(0.9)$ & $7.1(0.9)$ & $6.8(0.8)$ & $7.0(1.0)$ & 0.480 \\
\hline $\mathrm{HbA}_{1 \mathrm{c}}(\%)$ & $6.6(0.6)$ & $6.4(0.7) \dagger$ & $6.6(0.5)$ & $6.3(0.6) \dagger$ & 0.760 \\
\hline Plasma insulin (pmol/L) & $126(68)$ & $133(79)$ & $130(62)$ & $110(56)$ & 0.120 \\
\hline HOMA-IR & $5.8(3.0)$ & $6.1(3.6)$ & $5.6(2.7)$ & $4.9(2.6)$ & 0.256 \\
\hline Plasma AST (IU) & $26(25)$ & $23(11)$ & $21(11)$ & $20(14)$ & 0.787 \\
\hline Plasma ALT (IU) & $31(23)$ & $28(13)$ & $26(16)$ & $23(12)$ & 0.817 \\
\hline AST/ALT ratio & $0.8(0.2)$ & $0.9(0.2)$ & $0.9(0.3)$ & $0.9(0.4)$ & 0.560 \\
\hline Plasma $\gamma$-GT (IU) & $30(19)$ & $29(21)$ & $26(11)$ & $22(9)$ & 0.150 \\
\hline
\end{tabular}

Data are means (SD).

${ }^{*}$ Repeated-measures analysis of variance.

$\dagger P<0.05$ vs baseline.

ALT, alanine aminotransferase; AST, aspartate aminotransferase; BMI, body mass index; $\gamma$-GT, gamma-glutamyl transferase; $\mathrm{HbA}_{1 \mathrm{c}}$, glycated hemoglobin $A_{1 c}$; HDL, high-density lipoprotein; HOMA-IR, homeostatic model assessment of insulin resistance; LDL, low-density lipoprotein; MUFA, monounsaturated fatty acid.

In the latter three participants, tissue stiffness improved, reversing them to the $\mathrm{F} 0-\mathrm{F} 2$ stage.

At baseline, SWE measurements positively and significantly correlated with liver fat content $(\mathrm{r}=0.353, \mathrm{p}=0.030)$ and markers of liver function AST $(\mathrm{r}=0.480, \mathrm{p}=0.002)$, $\operatorname{ALT}(\mathrm{r}=0.388, \mathrm{p}=0.016)$, and $\gamma-\mathrm{GT}(\mathrm{r}=0.370, \mathrm{p}=0.022)$. No significant correlations were found between changes in tissue stiffness and changes in liver fat and markers of liver function after the dietary intervention.

\section{Anthropometrics and metabolic parameters}

Although the two dietary interventions were isocaloric, body weight decreased slightly in both groups during the 8-week treatments; however, this minimal reduction $(-1.2 \pm 1.3 \mathrm{~kg}$ with MUFA diet vs $-1.2 \pm 2.2 \mathrm{~kg}$ with multifactorial diet, $\mathrm{p}=0.95$ ) was not significantly different between the groups. Waist circumference did not change significantly during the intervention in the two groups (table 4).

No significant differences in AST, ALT, AST/ALT ratio and $\gamma$-GT were observed between baseline and end of intervention and between groups (table 4).

All participants had ALT levels in the normal range $(<50 \mathrm{IU} / \mathrm{L})$ but two subjects in the MUFA group and two in the multifactorial group. Adapting more restrictive cut-offs $^{27}$ to our laboratory upper limits, that is, using $30 \mathrm{IU} / \mathrm{L}$ for women and $40 \mathrm{IU} / \mathrm{L}$ for men, we found that six subjects in the MUFA group and three in the multifactorial group had ALT levels above the limits.

Fasting plasma glucose, plasma insulin, and HOMA-IR did not change significantly at the end of the interventions in either groups. Blood glucose control, as shown by $\mathrm{HbA}_{1 \mathrm{c}}$ levels, significantly improved after both the multifactorial diet $(6.57 \% \pm 0.50 \%$ vs $6.34 \% \pm 0.60 \%, \mathrm{p}=0.013)$ and the MUFA diet $(6.56 \% \pm 0.60 \%$ vs $6.37 \% \pm 0.67 \%$, $\mathrm{p}=0.024$ ) with no difference between groups (table 4 ). Fasting plasma concentrations of total, LDL, and HDL cholesterol and triglyceride did not change significantly during the intervention in either groups (table 4).

In the whole population, significant positive correlations were found between changes in liver fat and changes in $\gamma$-GT $(\mathrm{r}=0.470, \mathrm{p}=0.009)$, fasting plasma glucose $(\mathrm{r}=0.368$, $\mathrm{p}=0.023)$, and HbA1c $(\mathrm{r}=0.473, \mathrm{p}=0.008)$ (figure 2).

For all analyses, statistical significances did not differ when made for Intetion To Treat (ITT) or per protocol.

\section{CONCLUSIONS}

In this study, both a diet including different beneficial dietary items (multifactorial diet) and a diet high in MUFA (MUFA diet) were able to reduce liver fat content in patients with T2D, although the impact was significantly greater with the multifactorial diet. 
(A)

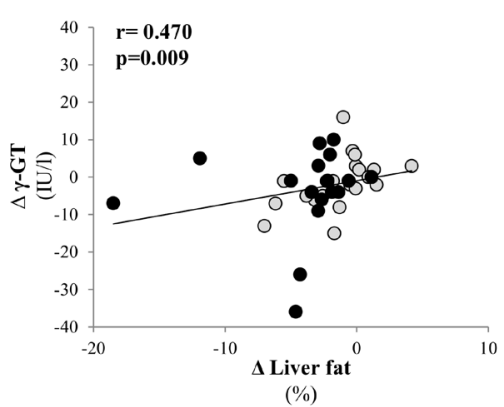

(B)

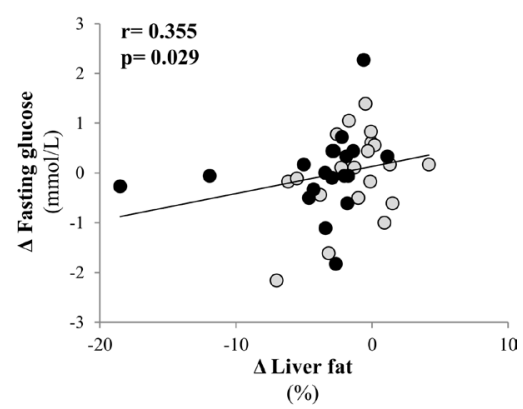

(C)

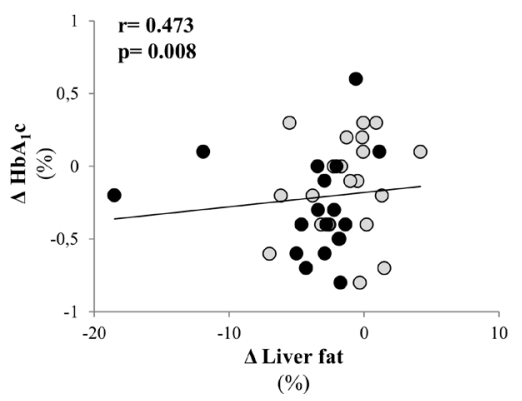

Figure 2 Spearman correlations between absolute changes after intervention in liver fat and $\gamma$-GT (A), fasting plasma glucose (B), and $\mathrm{HbA}_{1 \mathrm{c}}$ (C) levels. $\circ$, MUFA diet; $\bullet$, multifactorial diet; $\gamma$-GT, gamma-glutamyl transpeptidase; $\mathrm{HbA}_{10}$, haemoglobin $\mathrm{A}_{10}$; MUFA, monounsaturated fatty acid.

The multifactorial diet, independently of body weight change, induced a clinically relevant decrease $(40 \%)$ in liver fat content. This lowering effect was consistent in all participants.

The effect size of liver fat reduction achieved with our multifactorial diet is the highest obtained so far with any dietary or pharmacological intervention for treating liver steatosis in patients with T2D. In patients without diabetes, comparable outcomes were achieved with short-term dietary interventions either very low in carbohydrates or based on a substantial weight loss. ${ }^{28}{ }^{29}$ Both strategies would be very difficult to implement in clinical practice in the long term.

Our innovative approach, based on multiple small variations in the habitual diet, would be more feasible in the long term than any single very marked modification in energy or nutrient intake. This makes our dietary intervention a plausible therapeutic tool in clinical settings.

In consideration of the multiple changes characterizing the multifactorial diet, we are unable to identify which dietary components may have driven the beneficial effects of this approach. Its efficacy is likely related to the synergy of several dietary components targeting different pathophysiological mechanisms which may have a different relevance in individuals with diverse genetic and metabolic backgrounds. However, the most relevant changes in food choices induced by our multifactorial diet would impact primarily the intakes of polyphenols, other antioxidants and fiber; these can influence liver fat content through different mechanisms.
Data from human studies suggest that polyphenol supplements are able to increase energy expenditure and mitochondrial function. ${ }^{30}$ These pathways are crucial in the pathogenesis of liver fat. Moreover, it is possible that, when polyphenol intake is increased by the appropriate choice of natural foods included in a comprehensive dietary plan, they act synergistically and are able to boost MUFA and PUFA actions in promoting betaoxidation. Indeed, the ability of these dietary components to increase fatty acid oxidation has been proposed as the main mechanism through which they may be able to improve liver steatosis. ${ }^{31}{ }^{32}$ In addition, polyphenols would, at the same time, ensure mitochondrial efficiency by preserving them from the oxidative stress.

Polyphenols, other antioxidants and fibers included in our multifactorial diet could also have influenced liver fat through modifications of gut microbiota composition. ${ }^{33}$ The plausibility of this mechanism is also supported by results of a recent mechanistic trial in which dietary modifications induced changes in the gut microbiota composition significantly related to liver fat reduction. ${ }^{28}$ The high content in MUFA from EVOO, the low glycemic index of carbohydrate foods, the fiber and vitamins make the multifactorial diet reproduce the eating pattern of people living in Southern Italy in the 1950s-1960s. However, it also includes an amount of polyphenols resembling the healthy Nordic dietary pattern.

So far, to the best of our knowledge, few studies have evaluated the synergistic effect of multiple dietary changes on liver steatosis. The effects of the 
Cretan-Mediterranean dietary pattern were tested in a small group of non-diabetic people. This dietary intervention consisted in reducing the carbohydrate intake (33\%-36\% TEI) and increasing the intake of MUFA from EVOO (22\%-24\% TEI) and fiber ( $\approx 30 \mathrm{~g} /$ day $)$. The outcome of this dietary intervention was a $39 \%$ decrease in liver fat compared with a $7 \%$ liver fat reduction achieved with a low-fat diet. ${ }^{20}$ In contrast, in another study performed in obese people with liver steatosis diagnosed by ${ }^{1} \mathrm{H}-\mathrm{MRS}$, the Cretan diet induced a $20 \%$ reduction in liver fat, which was similar to that achieved with a low-fat diet as that recommended by the American Heart Association. ${ }^{21}$ The different outcomes observed in these two studies using similar dietary interventions is probably due to the longer follow-up of the study by Properzi $e t$ $a l,{ }^{21}$ which may have highlighted the potential of the lowfat diet in the long term.

In the aforementioned trials, the experimental diets were compared with control diets with a higher carbohydrate and a lower MUFA content. This makes it difficult to dissect the effects on liver fat content of carbohydrate reduction from those due to the higher MUFA intake. ${ }^{7}$

In our study, the amounts of fat and carbohydrates between the two dietary interventions were perfectly balanced. Therefore, the different outcomes on liver fat content were only accounted for by changes in the quality of fat and carbohydrate-rich foods.

As a secondary endpoint of the study, we evaluated changes in liver tissue elasticity measured by elastography, a reliable and accurate method for staging liver fibrosis. ${ }^{25}$ In this study, tissue stiffness positively correlated with liver fat content. It is possible that fat droplets in the hepatocytes may influence the architectural structure of the liver, thus altering tissue elasticity and changing the propagation time of the shear wave through the liver. ${ }^{34}$ Moreover, steatosis could interfere with liver stiffness through the induction of portal hypertension via modulation of nitric oxide and endothelin synthesis. ${ }^{35}$ We did not observe significant changes in tissue stiffness after either intervention, the very low prevalence of severe fibrosis in our cohorts at baseline giving little chance of observing such changes. Anyhow, three participants showed advanced fibrosis (F3-F4 stage, $>9.54 \mathrm{kPa}$ ) at baseline that reversed after diet, possibly leading to significant clinical benefits. However, the lack of biopsies does not allow ruling out of the possibility that the regression of steatosis may have affected the measurement of liver stiffness independent of changes in fibrosis.

Although $\mathrm{HbA}_{1 \mathrm{c}}$ significantly improved after both diets, we were unable to detect any difference in fasting plasma glucose and in indirect indices of insulin resistance between the two dietary interventions. This could be due to the very good blood glucose control of our participants, which may have blunted any further beneficial effect induced by the different diets. Moreover, HOMA-IR remains a poor index in patients with diabetes, and we had no direct measurements of insulin sensitivity (ie, glycemic clamp). In any case, these results clearly emphasize that the benefits achieved on liver fat content with the multifactorial diet were independent of metabolic control.

Our study has several strengths and some limitations. Possible limitations are related to the characteristics of the participants. They were in very good glycometabolic control, and about $30 \%$ of them were free of liver steatosis at baseline according to measurement by ${ }^{1} \mathrm{H}-\mathrm{MRS}$. Therefore, our conclusions cannot be extended to individuals with more severe disease. On the other hand, the results are strengthened by being observed in a population including also individuals without frank steatosis. Due to the lack of liver biopsies, we were unable to evaluate changes in inflammation and/or hepatocellular damage. More remarkably, we widened the range of dietary therapeutic options for NAFLD in T2D by a multifactorial approach based on food choices derived from different cultural backgrounds. This has high clinical relevance since compliance in the long term is the main drawback of dietary treatments in clinical settings. A wider range of food choices would facilitate the adhesion to healthy dietary plans also in everyday life and in different social, cultural and geographical contexts. In addition, favoring regional choices and substituting food of animal origin with plant-based foodstuffs, that is, preserving cultural diversity in eating habits, we found that our multifactorial diet would fully comply with the healthy diet models recommended to implement sustainable food systems. ${ }^{36}$

In conclusion, a multifactorial diet rich in beneficial dietary components, inspired by the Mediterranean diet from Southern Italy and including elements of other healthy dietary patterns, induced a $40 \%$ reduction of liver fat in people with T2D. Although studies with longer follow-up and tissue-related outcomes, in addition to liver fat, are needed, the multifactorial diet could be currently considered the diet with the best available evidence for preventing and treating NAFLD in patients with T2D.

\section{Author affiliations}

${ }^{1}$ Department of Clinical Medicine and Surgery, University of Naples Federico II School of Medicine and Surgery, Napoli, Campania, Italy

${ }^{2}$ IRCCS SDN, Napoli, Campania, Italy

${ }^{3}$ Institute of Biostructure and Bioimaging, National Research Council, Napoli, Campania, Italy

${ }^{4}$ Institute for Research on Population and Social Policies, National Research Council, Fisciano, Italy

Acknowledgements This study was supported by the Department of Clinical Medicine and Surgery of the Federico II University Hospital. Food for the study was kindly supplied by Lavazza, Torino, Italy (decaffeinated coffee); Pompadour Te' S.r.I, Bolzano, Italy (green tea); Coop. Nuovo Cilento s.c.r.l, San Mauro Cilento, Salerno, Italy, and Carapelli Firenze S.p.A, Italy (EVO0); Orogel S.p.A. Consortile, Cesena (FC), Italy (frozen vegetables); Conserve Italia Soc. coop. Agricola, San Lazzaro di Savena (BO), Italy (tomatoes and legumes); Aziende Campobasso S.r.l, Valenzano (BA), Italy (nuts).We thank Rosanna Scala (Department of Clinical Medicine and Surgery, Federico II University of Naples, Italy) for her professional linguistic support in the revision of the report.

Contributors GDP, CV, GA, GR, LB and AAR contributed to the design of the study and the analysis and interpretation of data. GDP, CV, MV and LB wrote the first draft of the report. GA, GR, MS, LB, and AAR provided relevant intellectual contribution to the development of the report. MV, GL, Al, and MT did the statistical analysis. SM, GC, MM, VB and MS were responsible for imaging (acquisition of images and results elaboration). PC, PM, and MS were responsible for laboratory (running of 
the laboratory and laboratory results). All authors provided substantial contribution to the acquisition of data, critically revised the report, and gave the final approval of the version to be submitted for publication. AAR is the guarantor of this work and, as such, had full access to all of the data in the study and takes responsibility for the integrity of the data and the accuracy of the data analysis.

Funding The authors have not declared a specific grant for this research from any funding agency in the public, commercial or not-for-profit sectors.

Competing interests None declared.

\section{Patient consent for publication Not required.}

Ethics approval The study protocol was reviewed and approved by the Federico II University Ethics Committee, and was conducted in accordance with the ethical principles outlined in the Declaration of Helsinki and the Good Clinical Practice guidelines. All participants provided written informed consent before recruitment (number of the approval: 16/17)

Provenance and peer review Not commissioned; externally peer reviewed.

Data availability statement Data are available upon reasonable request. AAR is the guarantor of this work and, as such, had full access to all of the data in the study (rivelles@unina.it).

Open access This is an open access article distributed in accordance with the Creative Commons Attribution Non Commercial (CC BY-NC 4.0) license, which permits others to distribute, remix, adapt, build upon this work non-commercially, and license their derivative works on different terms, provided the original work is properly cited, appropriate credit is given, any changes made indicated, and the use is non-commercial. See: http://creativecommons.org/licenses/by-nc/4.0/.

ORCID iDs

Marco Salvatore http://orcid.org/0000-0001-9734-7702

Angela Albarosa Rivellese http://orcid.org/0000-0002-6525-9780

\section{REFERENCES}

1 Stefan N, Häring H-U, Cusi K. Non-Alcoholic fatty liver disease: causes, diagnosis, cardiometabolic consequences, and treatment strategies. Lancet Diabetes Endocrinol 2019;7:313-24.

2 Bril F, Cusi K. Management of nonalcoholic fatty liver disease in patients with type 2 diabetes: a call to action. Diabetes Care 2017:40:419-30.

3 Promrat K, Kleiner DE, Niemeier HM, et al. Randomized controlled trial testing the effects of weight loss on nonalcoholic steatohepatitis. Hepatology 2010;51:121-9.

4 Vilar-Gomez E, Martinez-Perez Y, Calzadilla-Bertot L, et al. Weight loss through lifestyle modification significantly reduces features of nonalcoholic steatohepatitis. Gastroenterology 2015;149:367-78.

5 Della Pepa G, Vetrani C, Lombardi G, et al. Isocaloric dietary changes and non-alcoholic fatty liver disease in high cardiometabolic risk individuals. Nutrients 2017;9. doi:10.3390/ nu9101065. [Epub ahead of print: 26 Sep 2017]

6 Westerbacka J, Lammi K, Häkkinen A-M, et al. Dietary fat content modifies liver fat in overweight nondiabetic subjects. J Clin Endocrinol Metab 2005;90:2804-9.

7 Yki-Järvinen $\mathrm{H}$. Nutritional modulation of non-alcoholic fatty liver disease and insulin resistance. Nutrients 2015;7:9127-38.

8 Meisinger C, Rospleszcz S, Wintermeyer E, et al. Isocaloric substitution of dietary carbohydrate intake with fat intake and mridetermined total volumes of visceral, subcutaneous and hepatic fat content in middle-aged adults. Nutrients 2019;11. doi:10.3390/ nu11051151. [Epub ahead of print: 23 May 2019].

9 Bozzetto L, Prinster A, Annuzzi G, et al. Liver fat is reduced by an isoenergetic MUFA diet in a controlled randomized study in type 2 diabetic patients. Diabetes Care 2012;35:1429-35.

10 Errazuriz I, Dube S, Slama M, et al. Randomized controlled trial of a MUFA or Fiber-Rich diet on hepatic fat in prediabetes. J Clin Endocrinol Metab 2017;102:1765-74.

11 Bjermo H, Iggman D, Kullberg J, et al. Effects of n-6 PUFAs compared with SFAs on liver fat, lipoproteins, and inflammation in abdominal obesity: a randomized controlled trial. Am J Clin Nutr 2012;95:1003-12.

12 Schwimmer JB, Ugalde-Nicalo P, Welsh JA, et al. Effect of a low free sugar diet vs usual diet on nonalcoholic fatty liver disease in adolescent boys: a randomized clinical trial. JAMA 2019;321:256-65
13 Taskinen M-R, Söderlund S, Bogl LH, et al. Adverse effects of fructose on cardiometabolic risk factors and hepatic lipid metabolism in subjects with abdominal obesity. J Intern Med 2017;282:187-201.

14 Valtueña S, Pellegrini N, Ardigò D, et al. Dietary glycemic index and liver steatosis. Am J Clin Nutr 2006;84:136-42.

15 Cortez-Pinto $\mathrm{H}$, Jesus $\mathrm{L}$, Barros $\mathrm{H}$, et al. How different is the dietary pattern in non-alcoholic steatohepatitis patients? Clin Nutr 2006;25:816-23.

16 Rahman I, Biswas SK, Kirkham PA. Regulation of inflammation and redox signaling by dietary polyphenols. Biochem Pharmacol 2006;72:1439-52.

17 Annuzzi G, Bozzetto L, Costabile G, et al. Diets naturally rich in polyphenols improve fasting and postprandial dyslipidemia and reduce oxidative stress: a randomized controlled trial. Am J Clin Nutr 2014;99:463-71.

18 Bozzetto L, Annuzzi G, Pacini G, et al. Polyphenol-Rich diets improve glucose metabolism in people at high cardiometabolic risk: a controlled randomised intervention trial. Diabetologia 2015;58:1551-60.

19 Zelber-Sagi S, Salomone F, Mlynarsky L. The Mediterranean dietary pattern as the diet of choice for non-alcoholic fatty liver disease: evidence and plausible mechanisms. Liver Int 2017;37:936-49.

20 Ryan MC, Itsiopoulos C, Thodis T, et al. The Mediterranean diet improves hepatic steatosis and insulin sensitivity in individuals with non-alcoholic fatty liver disease. J Hepatol 2013;59:138-43.

21 Properzi C, O'Sullivan TA, Sherriff JL, et al. Ad libitum Mediterranean and low-fat diets both significantly reduce hepatic steatosis: a randomized controlled trial. Hepatology 2018:68:1741-54.

22 Vitale M, Masulli M, Cocozza S, et al. Sex differences in food choices, adherence to dietary recommendations and plasma lipid profile in type 2 diabetes - The TOSCA.IT study. Nutr Metab Cardiovasc Dis 2016;26:879-85

23 Lohman TM, Roche AF, Martorell R. Anthropometric standardization reference manual. Champaign, IL: Human Kinetics, 1988.

24 Weis J, Kullberg J, Ahlström H. Multiple breath-hold proton spectroscopy of human liver at 3T: relaxation times and concentrations of glycogen, choline, and lipids. J Magn Reson Imaging 2018;47:410-7.

25 Mancini M, Salomone Megna A, Ragucci M, et al. Reproducibility of shear wave elastography (swe) in patients with chronic liver disease. PLoS One 2017:12:e0185391-12.

26 Ferraioli G, Maiocchi L, Lissandrin R, et al. Accuracy of the ElastoPq Technique for the assessment of liver fibrosis in patients with chronic hepatitis C: a "real life" single center study. J Gastrointestinal Liver Dis 2016;25:333-5.

27 Prati D, Taioli E, Zanella A, et al. Updated definitions of healthy ranges for serum alanine aminotransferase levels. Ann Intern Med 2002;137:1-10.

28 Mardinoglu $\mathrm{A}$, Wu H, Bjornson $\mathrm{E}$, et al. An integrated understanding of the rapid metabolic benefits of a Carbohydrate-Restricted diet on hepatic steatosis in humans. Cell Metab 2018;27:559-71.

29 Gepner Y, Shelef I, Komy O, et al. The beneficial effects of Mediterranean diet over low-fat diet may be mediated by decreasing hepatic fat content. J Hepatol 2019;71:379-88

30 Most J, Timmers S, Warnke I, et al. Combined epigallocatechin3-gallate and resveratrol supplementation for $12 \mathrm{wk}$ increases mitochondrial capacity and fat oxidation, but not insulin sensitivity, in obese humans: a randomized controlled trial. Am J Clin Nutr 2016;104:215-27.

31 Bozzetto L, Costabile G, Luongo D, et al. Reduction in liver fat by dietary MUFA in type 2 diabetes is helped by enhanced hepatic fat oxidation. Diabetologia 2016;59:2697-701.

32 Hodson L, Gunn PJ. The regulation of hepatic fatty acid synthesis and partitioning: the effect of nutritional state. Nat Rev Endocrinol 2019;15:689-700.

33 Traber MG, Buettner GR, Bruno RS. The relationship between vitamin $C$ status, the gut-liver axis, and metabolic syndrome. Redox Biol 2019;21:101091.

34 Ferraioli G, Wong VW-S, Castera L, et al. Liver ultrasound elastography: an update to the world Federation for ultrasound in medicine and biology guidelines and recommendations. Ultrasound Med Biol 2018;44:2419-40.

35 Vincent MA, Montagnani M, Quon MJ. Molecular and physiologic actions of insulin related to production of nitric oxide in vascular endothelium. Curr Diab Rep 2003;3:279-88.

36 Willett W, Rockström J, Loken B, et al. Food in the Anthropocene: the EAT-Lancet Commission on healthy diets from sustainable food systems. Lancet 2019;393:447-92. 